\title{
VIBRATION CONTROL OF SUPER TALL BUILDINGS SUBJECTED TO WIND LOADS
}

\author{
Winston Chai and Maria Q. Feng \\ Department of Civil and Environmental Engineering, University of California, Irvine, \\ CA 92717, U.S.A.
}

\begin{abstract}
Excessive vibration due to wind loads is a major obstacle in design and construction of a super tall building. The authors recently introduced an innovative method for controlling the wind response of super tall buildings, which takes advantage of the so-called mega-sub structural configuration. Preliminary investigation was performed under the assumption that the wind load is a white noise and the building can be modeled as a shear structure. In this paper, a more reasonable tall building model (a cantilever beam) and a more realistic wind load model (a non-white stochastic process in time and space) are employed to design passive and hybrid mega-sub control systems and to examine the performance of such controlled buildings. Building vibration in both along-wind and across-wind directions is examined. The control parameters of the proposed systems, including the frequency ratio of the sub to the mega structures, the damping ratio of the sub structure, and the feedback gains of the actuator force, are studied and their optimal values are obtained. For comparison, a tall building without control and one with the conventional tuned-mass-damper control are also studied under the same load conditions. The significant cost-effectiveness of the proposed mega-sub systems is demonstrated in reducing the acceleration and deformation responses of tall buildings to wind loads, not only enhancing the safety of structure and its contents but also improving the comfort of occupants. (C) 1997 Published by Elsevier Science Ltd.
\end{abstract}

\section{INTRODUCTION}

As the urban population increases, construction of high-risc buildings presents a viable solution to the problems associated with urban society. Advances in construction technology and material science are making it possible to construct extremely tall buildings. This is exemplified by recent constructions of many high-rise buildings in Chicago, Tokyo, and Hong Kong. However, the safety of building structures and their contents as well as the comfort of occupants under such external forces as earthquakes and winds remains a significant engineering concern. For buildings with modest height, implementation of passive, active, or hybrid control devices offer a potential improvement in structural safety, performance of non-structural component, and human comfort, as these devices alter the dynamic characteristics of the structures to reduce structural response to external loads. For example, tuned mass damper systems have been applied to several buildings and were found to be effective in suppressing wind vibration. It is difficult, however, to introduce the conventional tuned mass damper system in tall or super tall buildings, since a heavier additional mass is required and a larger stroke must be accommodated in this case, thus raising significant safety concerns. Adding damping devices to the structure is another way to reduce the building vibration. Unfortunately, the structural characteristics common to most tall and super tall buildings, such as high shear rigidity and dominant bending deformation, tend to prevent the application of conventional damping devices.

A new method for controlling the response of tall buildings under severe external loads was recently introduced by Feng and Mita [1]. This method takes advantage of the so-called mega-sub structural configuration which is gaining popularity in design and construction of tall and super tall buildings, for example, the Bank of China at Hong Kong and Tokyo City Hall at Japan. A mega-sub building consists of two major components-a mega structure which is the main structural frame of the building and several sub structures each of which may contain several floors used for residential and/or commercial purposes. In original design, comparing to the design proposed, the sub structures are more rigid. Feng and Mita are the first to propose flexible sub structures such that the interaction between the mega and sub structures suppress the vibration of the entire building. The 
function of the sub structures is similar to that of the conventional mass damper in principle. The proposed mega-sub control system, however, is more advantageous than the conventional mass damper system. First of all, no additional mass is needed and the safety concern associated with the mass damper device for tall and super tall buildings is eliminated. Second and more important, the mass ratio between the sub and the mega structures is much higher (as high as $100 \%$ ) than that in the mass damper system (usually $1 \%$ ). It is this feature that makes the proposed control method much more effective. In an earlier study [1] the optimal paramcters for minimizing building response respectively to wind and earthquake load were identified, including the frequency ratio between the sub and the mega structures and the damping ratio of the sub structures. This mega-sub control is referred to as the passive mega-sub control since it is accomplished by properly designing the parameters related to dynamic properties of the structure. Later, Feng and Chai [2] proposed a hybrid mega-sub control concept in which an actively controlled actuator is added to the passively controlled mega-sub building to further reduce building response. The significantly high cost-effectiveness of the proposed passive and hybrid mega-sub control systems was demonstrated by the same study. However, in the previous studies, the wind and earthquake loads were modeled as a band-limited white noise, the mega-structure of the tall building was assumed to be of shear type, and the study was limited to the building vibration in the along-wind direction only.

In the present study, a cantilever beam is used to represent the mega structure which is a more reasonable model for a tall or super tall building in which bending is the dominant vibration mode. A more realistic wind load model is employed in which the turbulent wind speed is idealized as a non-white stochastic process in time and space. Consequently, the along-wind vibration is primarily generated by the additive excitations with non-linear colored noise (drag force), while the across-wind vibration is by the mutiplicative (parametric) excitations with non-linear colored noise (lift force). The across-wind vibration is due to the vortex shedding and depends on both the shedding frequency and the structural response [3-5]. The optimal parameters of the proposed passive and hybrid control systems, including the frequency ratio of the sub to the mega structures, the damping ratio of the sub structure, and the feedback gains of the actuator force, are obtained through both analytical and numerical methods. For comparison, the tall building without control and the one with the conventional tuned mass-damper control are also examined. In addition, the feasibility of the proposed systems is investigated through a numerical simulation of a representative building.

\section{TURBULENT WIND FORCE}

The wind speed profile along the vertical direction of a building is expressed as

$$
U(z, t)=\bar{U}(z)+u(z, t)
$$

where $\bar{U}(z)=$ mean wind speed at height $z, u(z, t)=$ fluctuating wind speed with a specified spectrum (non-white noisc).

The dominant along-wind wave length of the fluctuating wind speed is much larger than the major dimensions of the building in the plane under consideration.

The drag force in the along-wind $(x)$ direction can be expressed as [6-8]

$$
f_{x, i}(t)=\frac{1}{2} \rho C_{\mathrm{D}} A_{i}\left(U\left(z_{i}, t\right)-\dot{w}_{x, i}^{\mathrm{m}}\right)^{2}+\frac{\pi}{4} \rho C_{\mathrm{M}} A_{i} b\left(\dot{U}\left(z_{i}, t\right)-\ddot{w}_{x, i}^{\mathrm{m}}\right)
$$

where $\rho=$ air density, $b=$ lateral dimension of the building, $A_{i}=$ tributary area of the $i^{\text {th }}$ mass of the building in the along-wind direction, $C_{\mathrm{D}}=\mathrm{drag}$ coefficient, $w_{x, i}^{\mathrm{m}}=$ deflection of $i^{\text {th }}$ mass in the along-wind direction

In this equation, the interaction between the wind and the building has been taken into account. The second term of the equation accounts for the net force resulted from the accelerated air elements in the entire flow around the body, which is generally believed to be very small compared with the first term and thus can be neglected in the numerical computation, as advocated by Simiu and Scanlan [6]. 
The lift force acting on the building in the across-wind $(y)$ direction is induced by the vortex oscillation resulting from the wind flow shedding vortices in the wake of the building. The lift force depends on both the shedding frequency and the structural response [3-5], and it is modeled as $[4,5]$.

$$
\begin{aligned}
f_{y, i}(t) & =\frac{1}{2} \rho A_{i}^{\prime}\left(U\left(z_{i}, t\right)-\dot{w}_{x, i}^{\mathrm{m}}\right)^{2}\left[Y_{1}(k) \frac{\dot{w}_{y, i}^{\mathrm{m}}}{\left(U\left(z_{i}, t\right)-\dot{w}_{x, i}^{\mathrm{m}}\right)}+Y_{2}(k) \frac{\left(w_{y, i}^{\mathrm{m}}\right)^{2} \dot{w}_{y, i}^{\mathrm{m}}}{b^{2}\left(U\left(z_{i}, t\right)-\dot{w}_{x, i}^{\mathrm{m}}\right)}\right. \\
& \left.+J_{1}(k) \frac{w_{y, i}^{\mathrm{m}}}{b}+J_{2}(k) \frac{w_{y, i}^{\mathrm{m}}}{b} \sin \left(2 \omega_{\mathrm{s}} t\right)\right]
\end{aligned}
$$

where $A_{i}^{\prime}=$ tributary area of the $i^{\text {th }}$ mass in the across-wind direction, $w_{y, i}^{\mathrm{m}}=$ deflection of $i^{\text {th }}$ mass in the across-wind direction, $k=\omega_{1} b / \bar{U}(z)=$ reduced frequency, $\omega_{1}=$ fundamental frequency of the building, $\omega_{\mathrm{s}}=2 \pi \times \vartheta \vec{U}(z) / D=$ vortex shedding frequency, $\vartheta=$ Strouhal number and $D=$ effective dimension of the building.

The four terms in equation (3) respectively represent the linear and non-linear aeroelastic damping, aeroelastic stiffness, and parametric stiffness caused by vortex shedding. In order to verify this model, Goswami et al. $[4,5]$ performed a wind tunnel experiment with a circular cylinder to obtain the values of the coefficients $Y_{1}, Y_{2}, J_{1}$ and $J_{2}$.

The method to obtain the time history of the turbulent component of the wind speed was developed by Shinozuka and Deodatis [9] as follows:

$$
u(z, t)=\sqrt{2} \sum_{i=1}^{N_{1}} \sum_{j=1}^{N_{2}} \sqrt{2 S\left(\omega_{i}, k_{j}\right) \Delta \omega \Delta k}\left[\cos \left(\omega_{i} t+k_{j} z+\theta_{i j}\right)+\cos \left(\omega_{i} t-k_{j} z+\phi_{i j}\right)\right]
$$

where $S\left(\omega_{i}, k_{j}\right)=$ spectral density function of turbulent wind, $\Delta \omega=\omega_{\mathrm{u}} / N_{1}, \omega_{\mathrm{u}}=$ cut-off frequency, $\Delta k=k_{\mathrm{u}} / N_{2}, k_{\mathrm{u}}=$ cut-off wave number and $\theta_{i j}, \phi_{i j}=$ random phase angle, uniformly distributed between 0 and $2 \pi$.

The spectral density function of the following form was suggested by Davenport $[10,11]$ and used by Vaicaitis et al. [8] for the turbulent wind.

$$
S(\omega, k)=\frac{K^{\prime} \Phi^{2}}{2 \pi^{2}} \frac{|\omega|}{\left[1+\Phi^{2} \omega^{2} /(2 \pi \bar{U}(10))^{2}\right]^{4 / 3}} \frac{\varepsilon|\omega|}{\pi\left(\varepsilon^{2} \omega^{2}+k^{2}\right)}
$$

where $\Phi=$ scale of turbulence, $K^{\prime}=$ surface drag coefficient, $\varepsilon=$ constant $[\approx 0.006(\mathrm{~m} / \mathrm{s})]$ and $\bar{U}(10)=$ mcan wind specd at $10 \mathrm{~m}$ height above the ground

The spatial distribution of mean wind speed follows the logarithmic law.

$$
U(z)=\bar{U}(10) \frac{\ln \left(z / z_{0}\right)}{\ln \left(10 / z_{0}\right)}
$$

where $z_{0}=$ roughness length.

\section{ANALYTICAL MODELS}

The configuration of a conventional mega-sub building is illustrated in Fig. 1(a), where the main frame is the mega structure with several sub structures attached, while each sub structure usually contains several stories. For a tall or super tall building where bending is the dominant vibration mode, the mega structure should be modeled as a cantilever beam. This beam is further discretized as a multi-degree-of-freedom (MDOF) system. As pointed out earlier, the proposed mega-sub building should have the sub structures designed flexible so that the interaction between the mega and the sub structures can be used to suppress building vibration. A possible structural configuration which can achieve this design requirement by applying base isolation devices to sub structures is shown in Fig. 1(b). Since a sub structure is usually not slender, a shear-type structural model is appropriate for a sub structure. Therefore, the analytical model of a mega-sub controlled building can be obtained as shown in Fig. 2, together with the buildings controlled by other methods. 


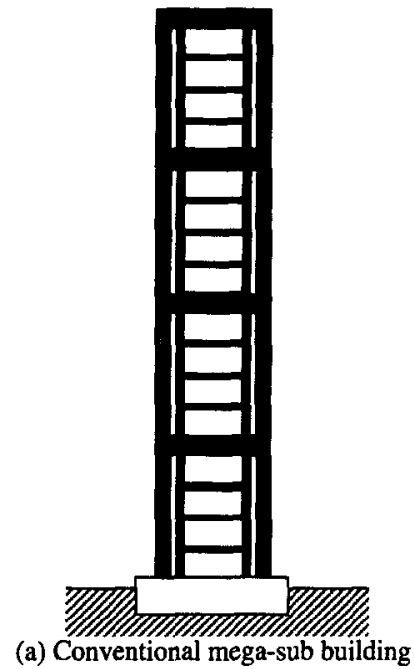

(a) Conventional mega-sub building

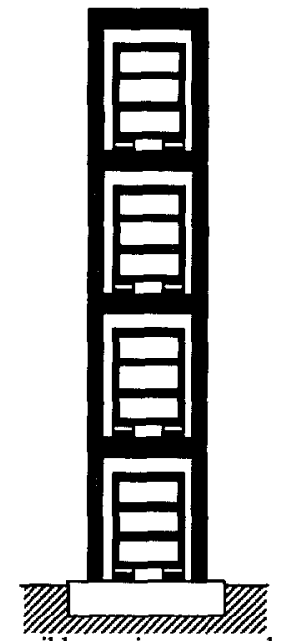

(b) A possible passive mega-sub controlled building with base-isolated sub structures

Fig. 1. Configuration of mega-sub building.

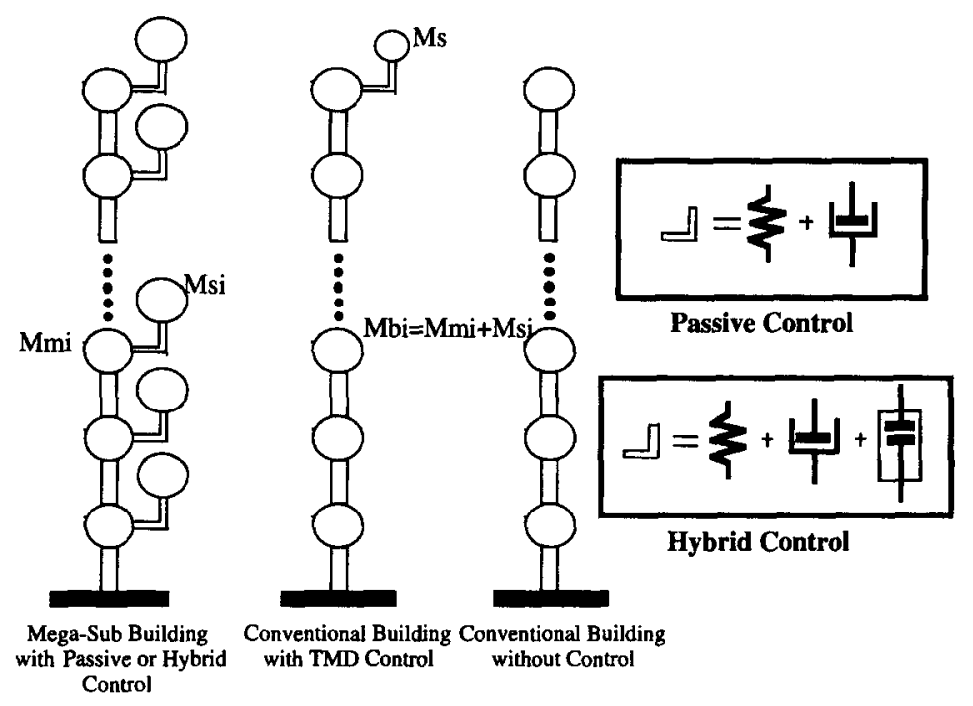

Fig. 2. Analytical model.

\section{Main structural frame}

The main structural frame is required to resist the external loads causing both translational and rotational deformation. For a uniform beam element, the stiffness matrix that relates the left end deformations and rotations, right end deformation and rotation to the corresponding shear and moments is given by

$$
\frac{E I}{L^{3}}\left[\begin{array}{rcrc}
12 & 6 L & -12 & 6 L \\
6 L & 4 L^{2} & -6 L & 2 L^{2} \\
-12 & -6 L & 12 & -6 L \\
6 L & 2 L^{2} & -6 L & 4 L^{2}
\end{array}\right]
$$

where $L=$ length of the beam element, $E=$ Young's modulus and $I=$ moment of inertia.

Assembling all the element stiffness matrices to a global system and moving all the freedoms corresponding to the translational deformation to the top portion of the matrix, the stiffness matrix, $K^{\mathrm{m}}$, of a cantilever beam can be formed. In order to simplify the calculation, a condensation operation [12] is performed, expressing the rotation-related 
terms in terms of the deformation terms. For this purpose, $K^{\mathrm{m}}$ is written as

$$
K^{\mathrm{m}}=\left[\begin{array}{ll}
K_{\mathrm{tt}} & K_{\mathrm{tr}} \\
K_{\mathrm{rt}} & K_{\mathrm{rr}}
\end{array}\right]
$$

where the subscriber $r$ denotes the rotation and $t$ the translational deformation. The condensed stiffness matrix is then

$$
K_{\mathrm{con}}^{\mathrm{m}}=\left[K_{\mathrm{tt}}\right]-\left[K_{\mathrm{tr}}\right]\left[K_{\mathrm{rr}}\right]^{-1}\left[K_{\mathrm{rt}}\right]
$$

The mass matrix before condensation is

$$
M^{\mathrm{m}}=\left[\begin{array}{cc}
{\left[m_{i}^{\mathrm{m}}\right]} & {[0]} \\
{[0]} & {\left[I m_{i}^{\mathrm{m}}\right]}
\end{array}\right]
$$

where $\left[m_{i}^{m}\right]=$ diagonal matrix consisting of masses of the mega structure or masses of the conventional building (without control or with tuned-mass-damper control) and $\left[\mathrm{Im}_{i}^{\mathrm{m}}\right]=$ diagonal matrix consisting of moment of inertial of the mega structure or moment of inertial of the conventional building (without control or with tuned-mass-damper control)

The condensed mass matrix is

$$
M_{\mathrm{con}}^{\mathrm{m}}=\left[m_{i}^{\mathrm{m}}\right]
$$

The uncondensed damping matrix is generated by the following equation [12] and the same equation can be applied to the condensed system.

$$
\left[C^{\mathrm{m}}\right]=\left[M^{\mathrm{m}}\right] \sum_{i} a_{i}\left(\left[M^{\mathrm{m}}\right]^{-1}\left[K^{\mathrm{m}}\right]\right)^{i} \quad i=1,2, \ldots, v
$$

where $\left[C^{\mathrm{m}}\right]=$ damping matrix, $v=$ number of modes,

$\xi_{j}=1 / 2 \omega_{j} \sum_{i} a_{i} \omega_{j}^{2 i}=$ damping ratio of the $j^{\text {th }}$ mode and $\omega_{j}=$ the $j^{\text {th }}$ natural frequency.

For the conventional building without control, the equation of motion is

$$
\left[M^{\mathrm{m}}\right]\left\{\ddot{X}^{\mathrm{m}}\right\}+\left[C^{\mathrm{m}}\right]\left\{\dot{X}^{\mathrm{m}}\right\}+\left[K^{\mathrm{m}}\right]\left\{X^{\mathrm{m}}\right\}=\left\{F^{\mathrm{m}}\right\}
$$

where $\left\{X^{\mathrm{m}}\right\}=$ deformation vector of building, $\left\{F^{\mathrm{m}}\right\}=$ external force vector.

The equation of motion for the condensed system is similar. For simplicity, the condensed mass, damping, and stiffness matrices are used to model the mega structure in this study.

\section{Mega-sub controlled building}

A sub mass is connected to each mega mass. The mass matrix of the mega-sub structure is expressed as

$$
\left[M^{\mathrm{ms}}\right]=\left[\begin{array}{cc}
{\left[M^{\mathrm{m}}\right]} & {[0]} \\
{[0]} & {\left[M^{\mathrm{s}}\right]}
\end{array}\right]
$$

where $\left[M^{\mathrm{m}}\right]=$ diagonal matrix consisting of masses of the mega structure and $\left[M^{\mathrm{s}}\right]=\mathrm{di}-$ agonal matrix consisting of masses of the sub structure.

The stiffness matrix of the mega-sub structure is

$$
\left[K^{\mathrm{ms}}\right]=\left[\begin{array}{cc}
{\left[K^{\mathrm{m}}\right]+\left[K^{\mathrm{s}}\right]} & -\left[K^{\mathrm{s}}\right] \\
-\left[K_{\mathrm{s}}\right] & {\left[K^{\mathrm{s}}\right]}
\end{array}\right]
$$

where $\left[K^{\mathrm{m}}\right]=$ stiffness matrix of the mega structure and $\left[K^{\mathrm{s}}\right]=$ diagonal stiffness matrix of the sub structures

The damping matrix of the mega-sub structure is expressed as

$$
\left[C^{\mathrm{ms}}\right]=\left[\begin{array}{cc}
{\left[C^{\mathrm{m}}\right]+\left[C^{\mathrm{s}}\right]} & -\left[C^{\mathrm{s}}\right] \\
-\left[C^{\mathrm{s}}\right] & {\left[C^{\mathrm{s}}\right]}
\end{array}\right]
$$

where $\left[C^{\mathrm{m}}\right]=$ damping matrix of the mega structure and $\left[C^{\mathrm{s}}\right]=$ diagonal damping matrix of the sub structures. 
For the mega-sub controlled building, the equation of motion is

$$
\left[M^{\mathrm{ms}}\right]\left\{\ddot{X}^{\mathrm{ms}}\right\}+\left[C^{\mathrm{ms}}\right]\left\{\dot{X}^{\mathrm{ms}}\right\}+\left[K^{\mathrm{ms}}\right]\left\{X^{\mathrm{ms}}\right\}=\left\{F^{\mathrm{ms}}\right\}
$$

where $\left\{X^{\mathrm{ms}}\right\}=$ deformation vector of the mega and sub building. For the passively controlled building,

$$
\left\{F^{\mathrm{ms}}\right\}=\left\{\begin{array}{c}
\left\{F^{\mathrm{m}}\right\}_{n \times 1} \\
\{0\}_{n \times 1}
\end{array}\right\}
$$

where $\left\{F^{\mathrm{m}}\right\}=$ external force vector and $n=$ number of mega masses while for the hybrid controlled building,

$$
\left\{F^{\mathrm{ms}}\right\}=\left\{\begin{array}{c}
\left\{F^{\mathrm{m}}\right\}_{n \times 1}+\left\{f_{\mathrm{a}}\right\}_{n \times 1} \\
-\left\{f_{\mathrm{a}}\right\}_{n \times 1}
\end{array}\right\}
$$

where $\left\{f_{\mathbf{a}}\right\}=$ actuator force vector.

\section{Conventional building controlled by tuned mass damper}

The tuned mass damper is usually installed on the top of the building. The mass matrix of the system is

$$
\left\{M^{\mathrm{md}}\right\}=\left[\begin{array}{cc}
{\left[M^{\mathrm{m}}\right]_{n \times n}} & \{0\}_{n \times 1} \\
\{0\}_{n \times 1}^{\prime} & m_{\mathrm{d}}
\end{array}\right]
$$

where the prime indicates transposition and $m_{\mathrm{d}}=$ mass of the mass damper. The stiffness matrix of the building is

$$
\left[K^{\mathrm{ms}}\right]=\left[\begin{array}{cc}
{\left[\left[K^{\mathrm{m}}\right]+\left\lceil K^{\mathrm{d}}\right]\right]} & -\left\{K^{\mathrm{d}}\right\} \\
-\left\{K^{\mathrm{d}}\right\}^{\prime} & k_{\mathrm{d}}
\end{array}\right]
$$

where $k_{\mathrm{d}}=$ stiffness of the mass damper.

$$
\begin{gathered}
\left\{K^{\mathrm{d}}\right\}=\left[\begin{array}{cc}
{[0]_{n \times n}} & \{0\}_{n \times 1} \\
\{0\}_{n \times 1}^{\prime} & k_{\mathrm{d}}
\end{array}\right] \\
\left\{\boldsymbol{K}^{\mathrm{d}}\right\}=\left\{\begin{array}{c}
\{0\}_{n \times 1} \\
k_{\mathrm{d}}
\end{array}\right\}
\end{gathered}
$$

The damping matrix of the building is

$$
\left[C^{\mathrm{ms}}\right]=\left[\begin{array}{cc}
{\left[\left[C^{\mathrm{m}}\right]+\left[C^{\mathrm{d}}\right]\right]} & -\left\{C^{\mathrm{d}}\right\} \\
-\left\{C^{\mathrm{d}}\right\}^{\prime} & c_{\mathrm{d}}
\end{array}\right]
$$

where $c_{\mathrm{d}}=$ damping of the mass damper

$$
\begin{aligned}
& {\left[C^{\mathrm{d}}\right]=\left[\begin{array}{ll}
{[0]_{n \times n}} & \{0\}_{n \times 1} \\
\{0\}_{n \times 1}^{\prime} & c_{\mathrm{d}}
\end{array}\right]} \\
& \left\{C^{\mathrm{d}}\right\}=\left\{\begin{array}{c}
\{0\}_{n \times 1} \\
c_{\mathrm{d}}
\end{array}\right\}
\end{aligned}
$$

For the conventional mega-sub building controlled by the mass damper, the equation of motion is

$$
\left[M^{\mathrm{md}}\right]\left\{\ddot{X}^{\mathrm{md}}\right\}+\left[C^{\mathrm{md}}\right]\left\{\dot{X}^{\mathrm{md}}\right\}+\left[K^{\mathrm{md}}\right]\left\{X^{\mathrm{md}}\right\}=\left\{F^{\mathrm{md}}\right\}
$$

where $\left\{X^{\mathrm{md}}\right\}=$ deformation vector of the building and mass damper

$$
\left\{F^{\mathrm{md}}\right\}=\left\{\begin{array}{c}
\left\{F^{\mathrm{m}}\right\}_{n \times 1} \\
0
\end{array}\right\}
$$
responses of the mega-sub building under the wind loads. In the present study, the target 
responses of a mega-sub building which must be controlled are the response acceleration of the sub structure and the deformation of the mega structure.

When a mega-sub building is simply modeled as a two-degree-of-freedom system with one mass representing the mega structure and the other the sub structure, two sets of the optimal values of the parameters (the frequency ratio between the sub structure to the mega structure $\beta_{\mathrm{opt}}$ and the damping ratio of the sub structure $h_{\text {sopt }}$ ) can be analytically found by minimizing the mean square value of each target response under the assumption that the wind force is a white noise [1]. However, for a more reasonable MDOF model under a more realistic non-white-noise wind load described in the above chapter, it is difficult to obtain the optimal parameters analytically.

In the present study, therefore, dealing with a discrete MDOF model of a mega-sub building, a numerical method is used to obtain the optimal frequency ratio $\beta_{\text {opt }}$ and optimal damping ratio $h_{\text {sop. }}$. Numerically solving the equation of motion, equations (17) and (18), subjected to the non-white-noise wind load described in equation (2), the peak target responses can be obtained for different sets of parameter $\beta$ and $h_{\mathrm{s}}$. Here, the displacement of the bottom mega mass and the acceleration of the top sub mass are chosen to be the target responses, since the base shear of the mega structure is the primary concern associated with the structural safety and the response acceleration of the sub structure at the top location is usually larger than that at other locations. These peak target responses can be plotted as functions of $\beta$ and $h_{\mathrm{s}}$. The values of $\beta$ and $h_{\mathrm{s}}$ which produce the minimum target response are the optimal values. It is noted that $\beta$ is defined as the ratio of the frequency of the sub structure to the fundamental frequency of the mega structure and $h_{\mathrm{s}}$ is defined as $c_{\mathrm{s}} /\left(2 m_{\mathrm{s}} \omega_{1}\right)$ where $\omega_{1}$ is the fundamental frequency of mega structure.

\section{DESIGN OF HYBRID CONTROLLED MEGA-SUB CONTROL}

As mentioned above, the optimal parameter values such as the frequency ratio and the damping ratio of the sub structures depend on the target responses of the building which need to be controlled. Therefore, it is impossible to find one set of optimal values which can achieve the minimum displacement of the mega structure and minimum acceleration of the sub structure simultaneously. This makes it difficult to design such a passively controlled mega-sub building. It is for this reason that the authors propose a hybrid-controlled mega-sub building in which an actively-controlled actuator is added to a passively controlled mega-sub building. In the passive control the frequency ratio and the damping ratio of the sub structures are set to the optimal values for minimizing the displacement of the mega structure, while in the active control the actuator feedback force is designed to minimize the acceleration of the sub structure.

The equations of motion for the hybrid controlled mega-sub building, equations (17) and (19), can be expressed as a state-space form

$$
\begin{aligned}
& \dot{X}(t)=A X(t)+B f_{\mathrm{a}}(t)+H F(t) \\
& \dot{Y}(t)=C X(t)+D f_{\mathrm{a}}(t)+E F(t)
\end{aligned}
$$

where

$$
\begin{aligned}
X(t)= & \left\{\left\{\dot{x}_{1}^{\mathrm{m}}(t) \sim \dot{x}_{n}^{\mathrm{m}}(t)\right\}_{1 \times n},\left\{\dot{x}_{1}^{\mathrm{s}}(t) \sim \dot{x}_{n}^{\mathrm{s}}(t)\right\}_{1 \times n},\left\{x_{1}^{\mathrm{m}}(t) \sim x_{n}^{\mathrm{m}}(t)\right\}_{1 \times n},\left\{x_{1}^{\mathrm{s}}(t) \sim x_{n}^{\mathrm{s}}(t)\right\}_{1 \times n}\right\}^{\mathrm{T}} \\
= & \text { state vector } \\
Y(t)= & \left\{\left\{x_{1}^{\mathrm{m}}(t) \sim \dot{x}_{n}^{\mathrm{m}}(t)\right\}_{1 \times n},\left\{x_{1}^{\mathrm{s}}(t)-x_{1}^{\mathrm{m}}(t)\right) \sim\left\{x_{n}^{\mathrm{s}}(t)-x_{n}^{\mathrm{m}}(t)\right)\right\}_{1 \times n}, \\
& \left.\left\{\ddot{x}_{1}^{\mathrm{m}}(t) \sim \ddot{x}_{n}^{\mathrm{m}}(t)\right\}_{1 \times n}\left\{\ddot{x}_{1}^{\mathrm{s}}(t) \sim \ddot{x}_{n}^{\mathrm{s}}(t)\right\}_{1 \times n}\right\}^{\mathrm{T}} \\
= & \text { output vector } \\
F(t)= & \text { wind force vector } \\
f_{\mathrm{a}}(t)= & -G X(t)=\text { actuator control force vector } \\
G= & \text { feedback control gain }
\end{aligned}
$$


and

$A, B, C, D, E, H=$ corresponding matrices deduced from equation of motion.

Applying the optimal control theory, the feedback control gain $G$ can be obtained to minimize the following objective index

$$
J=\int_{-\infty}^{\infty}\left\{Y^{\mathrm{T}}(t) Q Y(t)+f_{\mathrm{a}}^{\mathrm{T}}(t) R f_{\mathrm{a}}(t)\right\} \mathrm{d} t
$$

where $Q$ and $R$ are the weighting matrices whose elements are assigned according to the relative importance attached to the output variables and to the control force in the minimization procedure. Since the active control is used mainly for reducing the acceleration of the sub structures, those elements which correspond to the acceleration of the sub masses in the $Q$ matrix is heavily weighted.

\section{NUMERICAL SIMULATION}

In order to investigate the effectiveness and the feasibility of the proposed mega-sub control, responses of an example mega-sub building under wind loads are numerically simulated, and compared with the performance of a building without control and with the conventional mass-damper control. The building is assumed to be a $200-\mathrm{m}$ high steel structure covered by cylindrical facade with diameter of $40 \mathrm{~m}$. The total mass of the building is $m_{\mathrm{T}}=4.9 \times 10^{7} \mathrm{~kg}$. The mega structure is discretized as three concentrated masses, to each of which a sub mass is attached. The structural model and parameters applied for numerical simulation are shown in Fig. 3. The following four cases are simulated.

\section{Case 1: passively controlled mega-sub building}

The mass ratio of a sub mass to a mega mass is $u=1$ and the total mass is equally divided by the mega and sub masses. Hence, the mass of each mega structure $m_{\mathrm{m}}$ and the mass of each sub structure $m_{\mathrm{s}}$ are both equal to $m_{\mathrm{T}} / 6$. Figures 4 (a) and (b) show the peak target responses as functions of $\beta$ and $h_{\mathrm{s}}$. It should be pointed out that the response of the building in only the along-wind direction is simulated for this optimization purpose.

From Figs 4(a) and (b), it is found that the optimal parameter values are:

$\beta_{\text {opt }}=0.82$ and $h_{\text {sopt }}=0.16$ for minimizing the displacement of bottom mega mass; (30)

$\beta_{\text {opt }}=0$ and $h_{\text {sopt }}=0.35$ for minimizing the acceleration of top sub mass.

The frequency ratio of the sub structure to the mega structure and the damping ratio of the sub structure are set to the optimal values for minimizing the displacement of the bottom mega mass as shown in equation (30).

\section{Case 2: hybrid controlled mega-sub building}

An actuator is added between the top mega mass and the top sub mass of the building described in Case 1. The feedback control gain is designed to minimize the acceleration of the top sub mass.

Case 3: conventional building (without control)

The whole building is discretized to three concentrated masses with each mass $m_{\mathrm{b}}=m_{\mathrm{T}} / 3$.

\section{Case 4: tuned mass damper controlled conventional building}

A tuned mass damper is added on the top of the conventional building described in Case 3. The mass ratio of the damper mass to the building's total mass is $u=0.01$. The frequency ratio of the mass damper to the fundamental frequency of the building and the damping ratio of the mass damper are set equal to the optimal values $\beta_{\text {opt }}=0.9901$ and $h_{\text {sopt }}=0.0493$ that minimize the velocity of the building [2]. 

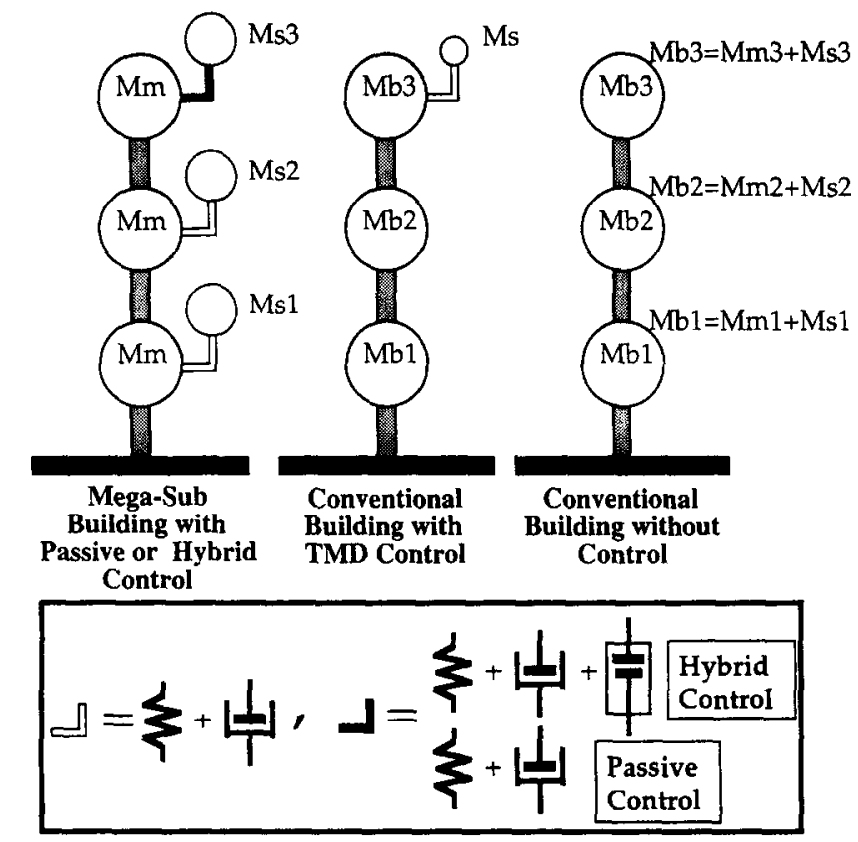

\begin{tabular}{|c|}
\hline Mega-Sub Bullding \\
\hline weight of mega mass \\
\hline 8000 (KN) \\
\hline weight of sub mass \\
\hline $8000(\mathrm{KN})$ \\
\hline El of mega structure \\
\hline $14^{*} 10^{\wedge} 10\left(\mathrm{KN}-\mathrm{m}^{\wedge} 2\right)$ \\
\hline fund. freq. of mega structure \\
\hline 2.22 (rad/sec) \\
\hline $\begin{array}{c}\text { damping ratios of all modes } \\
\text { for mega structure }\end{array}$ \\
\hline $1 \%$ \\
\hline $\begin{array}{c}\text { fund. freq. of mega-sub } \\
\text { structure }\end{array}$ \\
\hline 1.22 (rad/sec) \\
\hline
\end{tabular}

\begin{tabular}{|c|}
\hline Conven. Bulg. (w. MD) \\
weight of building mass \\
\hline 160000 (KN) \\
\hline weight of mass damper \\
\hline 4800 (KN) \\
\hline El of conven. bulg. \\
\hline $14^{*} 10^{\wedge} 10$ (KN-m²) \\
\hline fund. freq. of conven. bulg. \\
\hline 1.57 (rad/sec) \\
\hline $\begin{array}{c}\text { damping ratios of all modes } \\
\text { for conven. bulg. }\end{array}$ \\
\hline $1 \%$ \\
\hline $\begin{array}{c}\text { fund. treq. of conven. bulg. } \\
\text { with MD }\end{array}$ \\
\hline 1.50 (rad/sec) \\
\hline
\end{tabular}

Height: $\mathbf{2 0 0} \mathrm{m}$ Shape: Cylinder Diameter: $\mathbf{4 0} \mathrm{m}$

Fig. 3. Building model for numerical example.
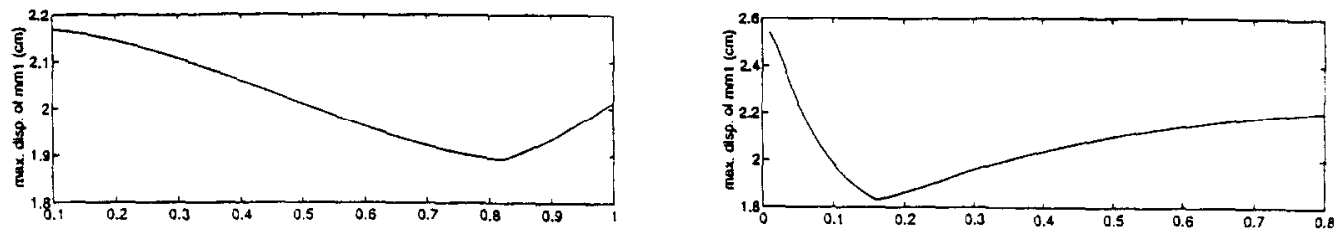

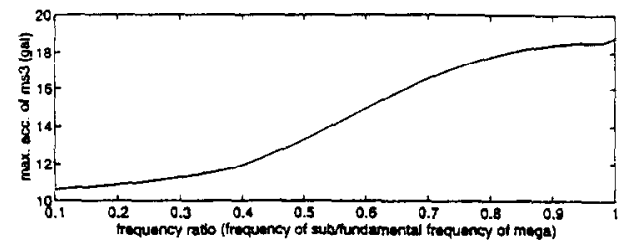

(a) with varied frequency ratio

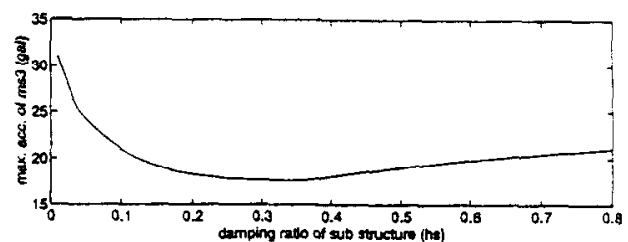

(b) with varied damping ratio

Fig. 4. Peak target response of passively controlled mega-sub building. 
Assuming the building is located at a suburban area, the following coefficient and parameter values are used to generate the wind force: $C_{\mathrm{D}}=1.8, K^{\prime}=0.03, \Phi=1200 \mathrm{~m}$, $z_{0}=0.3, \bar{U}(10)=21 \mathrm{~m} / \mathrm{s}, \vartheta=0.2, \rho=1.225 \mathrm{~kg} / \mathrm{m}^{3}$ and $D=40 \mathrm{~m} . Y_{1}, Y_{2}, J_{1}$ and $J_{2}$ at different heights are listed in Table 1.

The time histories of the target responses and the stroke (which is relative displacement between the top mega mass and the top sub mass) in both along-wind and across-wind directions are computed and shown in Figs 5 and 6, among the buildings with different control systems described in the above four cases. The trajectories of the target responses and the stroke are respectively shown in Figs 7(a), (b) and (c). From Figs 5(a) and (b), it is found that the displacement of the bottom mega mass (which is proportional to the base shear) and the acceleration of the top sub mass are significantly reduced by the passive mega-sub control, which cannot be achieved by the conventional mass damper control. Figures 6(a) and (b) demonstrate that the hybrid mega-sub control can further reduce the acceleration of the top sub mass while keeping the displacement of the bottom mega mass within a small range. In addition, the peak actuator force needed for the hybrid mega-sub

Table 1. Coefficient values for fluctuation wind speed

\begin{tabular}{rrllr}
\hline Height $(\mathrm{m})$ & $Y_{1}$ & \multicolumn{1}{c}{$Y_{2}$} & \multicolumn{1}{c}{$J_{1}$} & \multicolumn{1}{c}{$J_{2}$} \\
\hline 200.00 & 8 & 0.1 & 0 & 800 \\
133.33 & 20 & 0.00001 & 0 & 1500 \\
66.67 & 8 & 0.00001 & 0 & 2500 \\
\hline
\end{tabular}

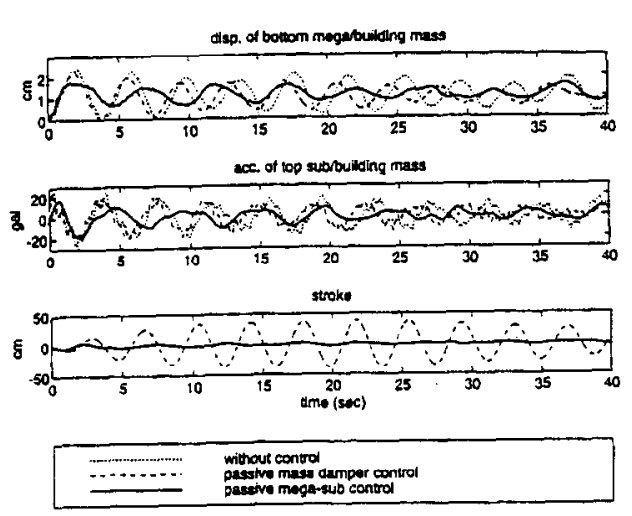

(a) along-wind direction

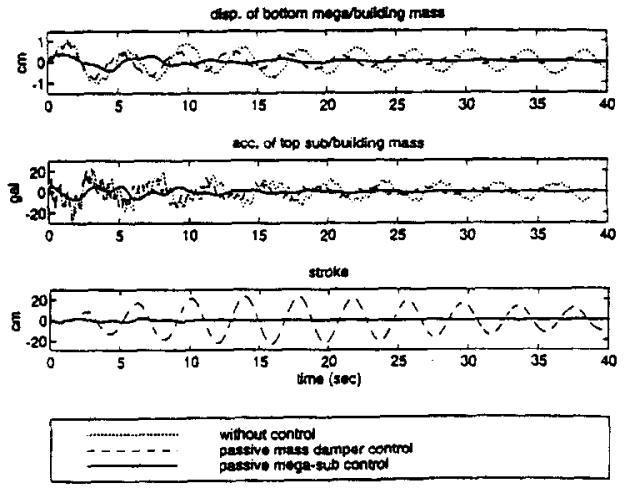

(b) across-wind direction

Fig. 5. Response comparison (non-control, mass damper and passive mega-sub control).
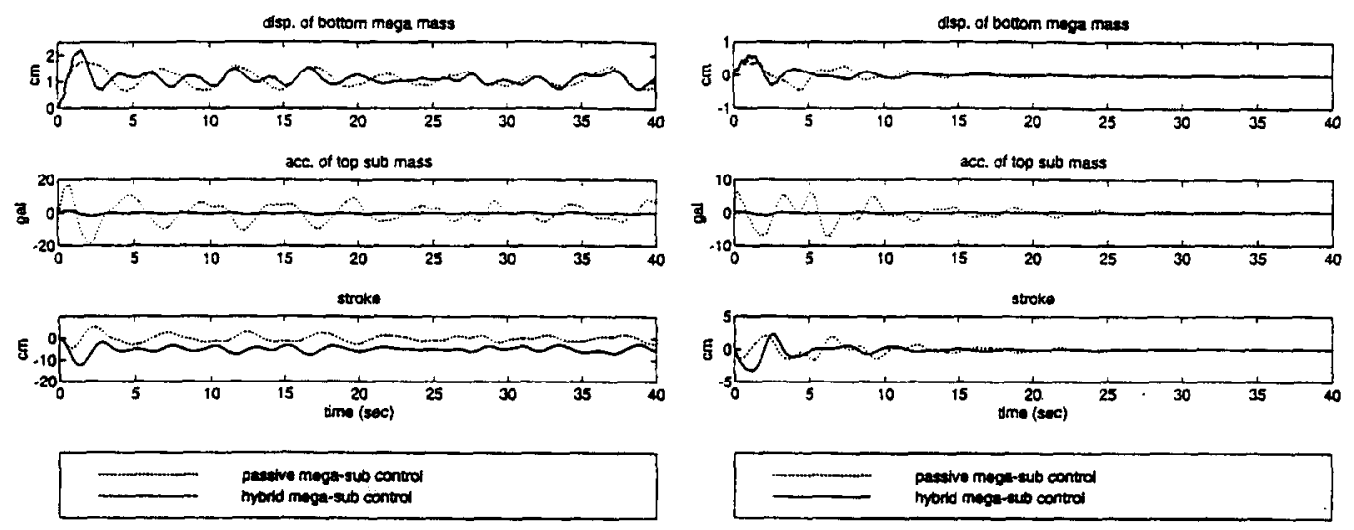

(a) along-wind direction

(b) across-wind direction

Fig. 6. Response comparison (passive and hybrid mega-sub control). 


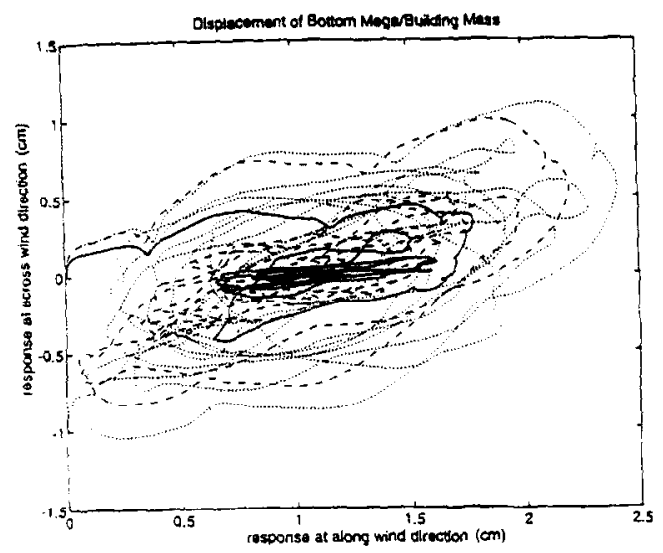

(a) displacement of bottom mega mass

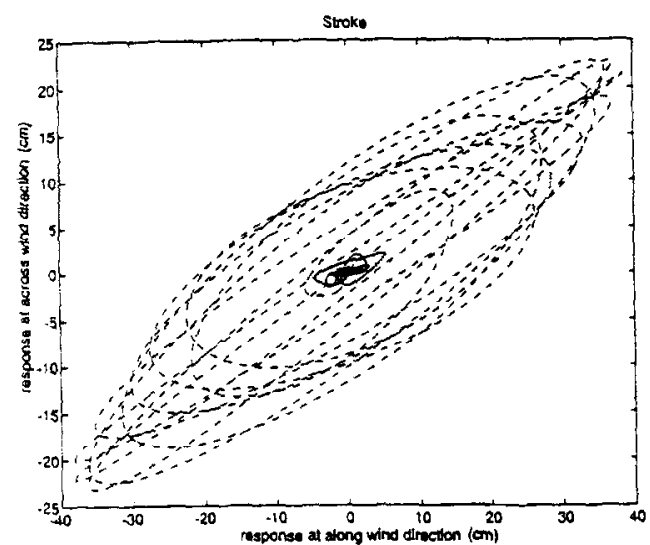

(c) stroke

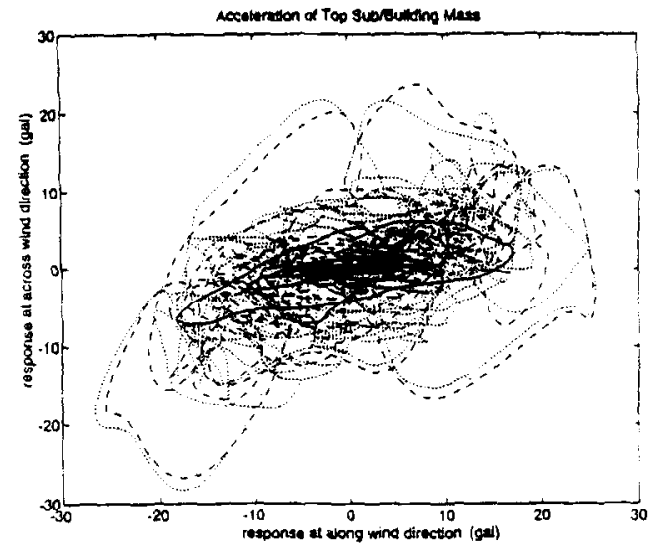

(b) acceleration of top sub mass

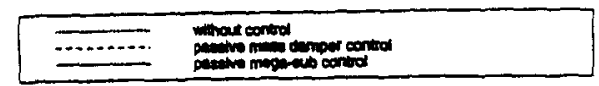

Fig. 7. Comparison of response trajectories.

control is only $0.78 \%$ of the total building weight. This, together with the small amount of stroke indicated in the time histories, make the proposed hybrid control feasible and significantly practical. Also, the amplitude of the building responses to initial excitation at the across-wind direction decays as time proceeds. The self-excited and self-limited characteristics of the building at the across-wind direction is thus clearly demonstrated. It is noted that the interaction between the building responses and wind at across-wind direction required the initial conditions of building to initiate the process, and this character is shown in equation (3). On the other hand, it is pointed out that, theoretically, the building responses will diverge when the wind force exceeds a certain critical value due to instability. However, this critical value is very large and hence it is unlikely to occur in reality.

\section{CONCLUSION}

Innovative mega-sub control systems with or without actuators are designed to reduce wind responses in super tall buildings, using a non-white-noise stochastic process in time and space to model wind speed and a cantilever beam to model the building. The optimal values of passive control parameters are generated by a numerical method, and the optimal feedback control gain for the actuator in the hybrid control case is obtained on the basis of the optimal control theory.

Through simulation of building responses in both along-wind and across-wind directions using different control methods, including the proposed mega-sub control and the conventional mass damper control, it is demonstrated that (1) the mega-sub control is much more 
effective than the conventional mass damper control in reducing target responses such as deformation of the mega structure and acceleration of the sub structure; and (2) with the help of an actuator, a certain target response can be further reduced without requiring much power and space for stroke. Besides its significant effectiveness, the proposed control method is feasible and practical since the required optimal structural parameters are easy to achieve in the actual design and construction and, in addition, the required control force and stroke of the actuator are minor.

Acknowledgements-This work was supported by the Career Award of the National Science Foundation and monitored by Dr E. Sabadell.

\section{REFERENCES}

1. M. Q. Feng and A. Mita, Vibration of tall buildings using mega-sub configuration. ASCE Journal of Engineering Mechanics 121, (1995).

2. M. Q. Feng and W. Chai, Hybrid-controlled mega-sub buildings. Submitted to ASCE Journal of Engineering Mechanics (1995).

3. K. Billah, A study of vortex-induced vibration. Ph.D. Dissertation, Princeton University (1989).

4. I. Goswami, R. Scanlan and N. Jones, Vortex-induced vibration of circular cylinders. I: Experimental data. ASCE Journal of Engineering Mechanics 119, 2270-2287 (1993).

5. 1. Goswami, R. Scanlan and N. Jones, Vortex-induced vibration of circular cylinders. II: New model. $A S C E$ Journal of Engineering Mechanics 119, 2288-2302 (1993).

6. E. Simiu and R. Scanlan, Wind Effect on Structure, second edition. Wiley, New York (1986).

7. R. Vaicaitis, M. Shinozuka and M. Takeno, Parametric study of wind load on structure. Journal of Structural Division 99, 453-468 (1973).

8. R. Vaicaitis, M. Shinozuka and M. Takeno, Response analysis of tall buildings to wind loading. Journal of Structural Division 101, 585-600 (1975).

9. M. Shinozuka and G. Deodatis, Stochastic process models for earthquake ground motion. Probabilistic Engineering Mechanics 3, 114-123 (1988).

10. A. G. Davenport, The application of statistical concepts to wind loading of structures. Proceedings, Institution of Civil Engineers, Vol. 19, pp. 449-472 (1961).

11. A. G. Davenport, The response of slender line-like structures to a gusty wind. Proceedings, Institution of Civil Engineers, Vol. 23, pp. 389-408 (1962).

12. M. Paz, Structural Dynamics, third edition. Van Nostrand Reinhold, New York (1991). 